\title{
Topological model of homoclinic chaos in a glow discharge
}

\author{
Thomas Braun, Ricardo R.B. Correia, and Nara Altmann \\ Instituto de Física - Universidade Federal do Rio Grande do Sul, Caixa Postal 15051, 91501-970 Porto Alegre - RS, Brazil
}

(Received 16 May 1994)

\begin{abstract}
We perform a topological analysis of homoclinic chaos in an experimental dynamical system: the glow discharge (GD). The geometrical model accounting for the stretching and folding mechanisms in the GD attractor is the horseshoe template. The presence of a homoclinic orbit induces a structure on this template, allowing us to predict the qualitative dynamical behavior as the GD approaches homoclinicity.
\end{abstract}

PACS number(s): 05.45.+b, 52.80.Hc

The knowledge that deterministic equations of motion may have complex nonperiodic solutions was originated by Poincaré as he introduced the homoclinic tangle [1]. For instance, considering a three variable dynamical system in its corresponding three-dimensional (3D) phase space, the homoclinic tangle is formed when the stable and unstable manifolds emanating from a saddle type periodic orbit $(\gamma)$ present in the flow approach intersection. The saddle nature of this cycle forces the phase space trajectory either to approach $\gamma$ or to diverge from it. Thus, in the flow, the stable manifold $W^{s}(\gamma)$ is the collection of all solutions that approach the unstable orbit $\gamma$ while the unstable manifold $W^{u}(\gamma)$ is the collection of all solutions diverging from $\gamma$. The tangency (intersection) of these stable and unstable manifolds in the flow defines a very elusive kind of orbit: as it belongs to both manifolds this orbit must approach the saddle cycle for $t \rightarrow \pm \infty$. This kind of orbit is called a homoclinic orbit and its presence in the flow tends to promote erratic behavior, usually termed homoclinic chaos [2].

Nowadays, the characterization of chaos from the analysis of observed chaotic data mainly follows two general approaches [3]. The metric approach extracts metric invariants from the dynamics and the topological approach is concerned with invariants obtained from the topology of the strange attractor. Both metrical and topological invariants are independent of coordinate changes, but only topological invariants remain invariant under smooth parameter changes. The central object in the topological approach is the "template" [4-6], which is essentially a geometrical model of the chaotic dynamics. We are interested in the topological aspects of the flow originated by the reported $[7,8]$ homoclinic behavior in the glow discharge (GD). In this sense the aim of this paper is twofold: first, to derive the template underlying the chaotic dynamics of the GD and second, to realize the structure induced on the template by the presence of a homoclinic orbit in the dynamics. From this we can establish a topological model which enables us to predict the dynamics of the GD as the control parameter is changed. This prediction concerns, for instance, selection rules for the symbolic sequence of the orbits. Our analysis enables us to address two important issues of the topological characterization of chaos: the pruning [9] of unstable periodic orbits and the change of the template induced by a control parameter variation.
An experimental system will never reach a homoclinic orbit because of unavoidable small fluctuations (noise). But in spite of this, a complex dynamical behavior (chaos) may be expected when the system in its phase space description is near a homoclinic orbit, as was demonstrated by Gavrilov and Shilnikov [10]. Experimental systems in which the dynamics is strongly influenced by the proximity of a homoclinic orbit, as analyzed in Ref. [10], are, for example, a laser with a saturable absorber [11] and a GD [7]. In the latter, while approaching the homoclinic orbit, there is evidenced a rich bifurcation sequence known as an alternating periodic-chaotic (APC) sequence. This is made up of self-induced oscillations on the GD current displaying an alternation of periodic and chaotic patterns of large and small amplitude oscillations. The experimental results presented in this paper are obtained from a dc-excited GD, the setup of which can be found elsewhere [7]. The operating conditions are such that the GD presents an APC sequence induced by the change of a control parameter (voltage feeding the GD). For a typical bifurcation diagram see Ref. [8].

In order to perform the topological analysis, we consider the $C^{(2)}$ chaotic window of the APC sequence (see Ref. [7] for the meaning of the notation) and now analyze thoroughly the structure of the corresponding phase space description. A 3D reconstructed phase space is obtained from the GD current time evolution $I(t)$ applying the time delay embedding with $X=I(t), Y=I(t+\tau)$, $Z=I(t+2 \tau)$, and $\tau=0.96 \mu \mathrm{s}$. At the center of Fig. 1 is shown an $X Y$ projection of the attractor corresponding to the chaotic $C^{(2)}$ regime; the flow is clockwise oriented. The general feature of the dynamics in phase space consists [8] of an unstable spiraling behavior [near the central hole in the diagram where a saddle cycle $(\gamma)$ is located] connected to a reinjection mechanism. Thus, regarding the stable and unstable manifolds of the saddle cycle, a chaotic trajectory approaches the cycle along $W^{\boldsymbol{s}}(\gamma)$, then it begins to escape spiraling outward along $W^{u}(\gamma)$, and finally the reinjection mechanism, which is due to nonlinearities present in the flow, brings the trajectory again very near to the saddle cycle.

We take advantage of the fact that, according to Fig. 1 , inside the attractor there is a hole and define a rotation axis centered in this hole and parallel to the $Z$ axis. Next, we consider a Poincaré section plane $\Sigma$ with one of its borders coincident with the rotation axis and then 


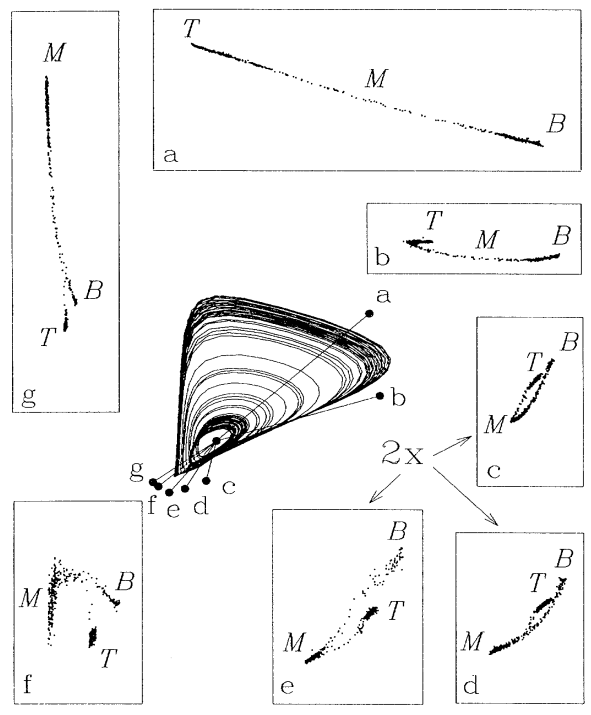

FIG. 1. Chaotic phase space portrait of the glow discharge (at the center) surrounded by cross sections performed in the flow along the lines $a, b, \ldots, f, g$. Cross sections $c, d$, and e are enlarged $2 \times$.

sweep $\Sigma$ a full revolution through the attractor. In the phase portrait of Fig. 1 the lines labeled with $a, b, \ldots, f, g$ indicate transversal cross sections obtained from the flow proceeding in this way. The intersection points of the flow with the cross sections are displayed in the boxes surrounding the attractor's projection. In all of them, we realize that the intersections of the chaotic trajectory with the cross sections scatter along a line, indicating that the attractor is almost a surface. This usually happens in dissipative systems. As in the Duffing and Lorenz attractors [2], we conjecture that this surface is bounded by an unstable manifold, in our case, $W^{u}(\gamma)$. Therefore, considering the Poincaré section (a) of Fig. 1, the saddle cycle $\gamma$ is reduced to a saddle fixed point $p$ of the 2D map defining the next return to the same section. The stable and unstable manifolds of $p, W^{s}(p)$ and $W^{u}(p)$, are formed by the intersections of $\Sigma$ with, respectively, $W^{s}(\gamma)$ and $W^{u}(\gamma)$. Thus, the straight segment $T B$ of Fig. 1(a) lies on $W^{u}(p) ; p$ is located to the right of $B$. The map is considered to be in the form $f(x, y ; \mu)$. We assume further that the dynamics is such that the manifolds of $p$ are about to develop a tangency (i.e., a homoclinic point) as in Ref. [10]. The parameter $\mu=0$ corresponds to the homoclinic tangency whereas the values $\mu>0$ quantify the "distance" to this tangency.

Now we want to follow the iterates of the segment $T B$ [Fig. 1(a)] until they intersect it again. For this purpose, we analyze the complete sequence of Poincaré sections, beginning from Fig. 1(a). It is possible to identify clearly the stretching and folding of the attractor's surface. To better realize this geometry, we marked in cross section (a) three points: one at the top $(T)$, other in the middle $(M)$, and the third at the bottom $(B)$. Following these points' locations at the succeeding cross sections, we see that, after one revolution, the initial straight interval $T B$ is transformed into a line segment that resembles a horseshoe. This new conformation of the segment $T B$ also lies on $W^{u}(p)$, because the iterates of the initial straight segment, under the action of $f$, must remain on $W^{u}(p)$ for the reason that the manifold is invariant. Therefore, this transformation may be summed up representing the incipient tangling of $W^{u}(p)$ in the creation of a homoclinic tangency [2], as shown in Fig. 2. There, the straight segment $T B$ corresponds to section (a) of Fig 1 . The saddle fixed point $p$ is located at the origin. After iterating once this segment, the segment $T B$ appears as the stretched and folded bold line on $W^{u}(p)$ in Fig. 2, following the bending of $W^{u}(p)$ while the latter approaches $W^{s}(p)$ and $p$. As the GD is strongly dissipative, the segment $T B$ is mapped onto its original straight position after just one iteration. This recurrent behavior, envolving the operations of stretching and folding, is responsible for the chaotic dynamics and thus for the geometrical conformation of the chaotic attractor. Unstable periodic orbits (knots) that are embedded within the chaotic attractor [9] are then constrained by this geometric structure and it is the goal of a topological analysis to establish a geometrical model that captures this intricate topological organization. The result is the "knotholder" or template $[4-6]$.

Usually a template is obtained [6] by first searching for unstable periodic orbits in the attractor and then determining topological invariants (linking numbers, relative rotation rates, etc.) which are related to the knotting and linking of the unstable periodic orbits on the attractor. On the basis of these topological invariants the template is identified. We instead capture the global organization of the unstable periodic orbits by analyzing their support: the chaotic attractor. A template may be constructed following a complete revolution of an attractor's cross section [12]. The succeeding cross sections evidence the stretching and folding mechanisms of the attractor and how the flow is reinjected into itself. That is essentially what the template has to show. We begin with cross section (a) of Fig. 1 and consider the segments $T M$ and $M B$ associated to two ribbons; they will be branches of the template. Now the $T M$ ribbon is half-twisted and then both, after undergoing a lateral expansion, are glued together at a line called the branch

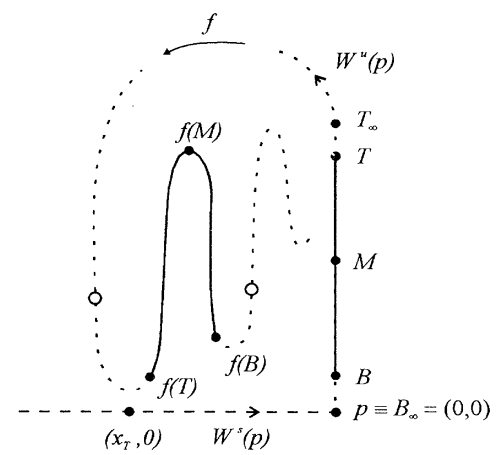

FIG. 2. Geometry for the creation of a homoclinic tangency. 
line. Finally, the branch line is identified with the initial segment $T B$. The geometrical implementation of this transformation results in the "horseshoe template" [5] displayed in Fig. 3. Thus, the template is formed by the projection of the flow along the stable manifold, as described in Ref. [5]. Orbits of the flow are then projected onto the template preserving their topological organization. Therefore, more precisely, the template is a semiflow (because inverse orbits are not unique at the branch line) on a branched 2D manifold. The outlined procedure to obtain the template has already been applied, for instance, in a numerical investigation of the Duffing oscillator [12], and in the experimental study of a modulated laser [13]. Both systems are forced, which facilitates the topological analysis, while the GD is subject to no external forcing. The template we obtained is also confirmed [14] by the knot analysis of the unstable periodic orbits identified in the GD data, in the same way that the horseshoe template of a laser with saturable absorber is derived [15].

In Fig. 1(a), the location of point $M$ in $T B$ is not arbitrary. $M$ is the frontier between two regions in the phase space. One $(T M)$ is associated to the reinjection mechanism, whereas the other one $(M B)$ corresponds to the looping around the saddle cycle. These two distinct processes enable us to codify the GD dynamics with two symbols, $R$ (reinjection) or $L$ (looping), in accordance whether the phase space flow crosses $T M$ or $M B$. The homoclinic orbit corresponds in phase space to one reinjection loop followed by an infinite number of loopings around the saddle cycle $[7,8]$ (this orbit is represented on the template of Fig. 3) and therefore the symbolic sequence $R L^{\infty}$ will be associated to it. The same result is also obtained from $1 \mathrm{D}$ return maps [8].

The template of Fig. 3 corresponds to the homoclinic tangency $(\mu=0)$. In this situation the chaotic attractor is not yet hyperbolic [2] due to the coincidence between the stable and unstable directions at the tangency point. The complete hyperbolic regime sets in after the occurrence of the last tangency between $W^{s}(p)$ and $W^{u}(p)$, when then all possible periodic orbits are described by the symbolic dynamics on a complete binary ( $R$ or $L$ ) tree. For parameter values $\mu$ prior to the homoclinic tangency, the GD dynamics is likely not to be hyperbolic

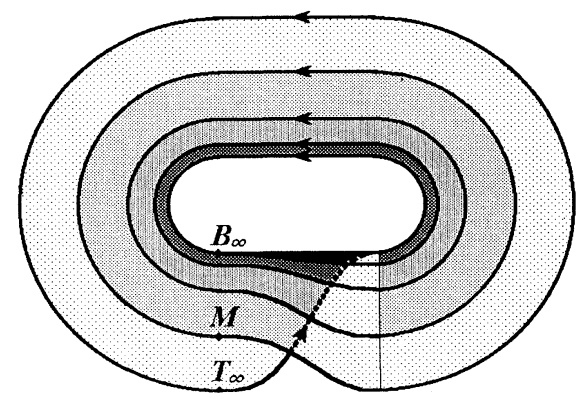

FIG. 3. Horseshoe template supporting an $R L^{\infty}$ homoclinic orbit. and therefore not all possible combinations of $R$ and $L$ have a counterpart in the observed periodic orbits (i.e., they are "pruned"). As $\mu$ assumes positive values, the GD departs more from hyperbolicity and therefore to account for the associated pruning process, we say that the unstable periodic orbits of the attractor are constrained to only a subset of the template of Fig. 3. As $\mu \rightarrow 0$, the qualitative dynamics of the GD is modeled assuming that a larger extent of the template is visited by the orbits, permitting a larger amount of combinations between $R$ and $L$. Based on experimental observations, we implemented this idea in Fig. 4 for the $C^{(1)}$ and $C^{(2)}$ chaotic windows of the APC sequence. According to the template construction, in Fig. 4 are represented the stretching and folding mechanisms for the $C^{(1)}$ (white drawing) and the $C^{(2)}$ (gray drawing) attractors. It is important to notice that, by action of $f$ (see Fig. 2), the fold of the segments $T_{1} B_{1}$ and $T_{2} B_{2}$, corresponding, respectively, to the $C^{(1)}$ and $C^{(2)}$ windows, is asymmetric because $f\left(T_{n} M\right)>f\left(M B_{n}\right)$ ( $n$ is the superscript of $\left.C^{(n)}\right)$. For the $C^{(2)}$ regime this is evidenced in Fig. $1(\mathrm{~g})$. The inequality is still larger for the $C^{(1)}$ regime and it will become smaller for $C^{(n)}$ regimes when $n \rightarrow \infty$. This is in agreement with the fact that, according to Fig. 2, the "parabolalike" bendings of $W^{u}(p)$ depart exponentially from $W^{s}(p)$ as $\mu$ rises (i.e., $n \rightarrow 0$ ) [2] originating the growing inequality $f\left(T_{n} M\right)>f\left(M B_{n}\right)$. On the other hand, at the homoclinic tangency $(\mu=0 ; n=\infty)$, we have $f\left(T_{\infty} M\right)=f\left(M B_{\infty}\right)$ which implies that in Fig. 2 the location of $f\left(T_{\infty}\right)$ will then be $\left(x_{T}, 0\right)$. The template (Fig. 3) corresponds exactly to this situation. The $R L^{\infty}$ homoclinic orbit splits this template into $P^{(n)}$ strips $(n=0,1,2, \ldots, \infty$; they correspond to the shadowed areas delimited by the homoclinic orbit in Fig. 3 and are also represented at the left of Fig. 4). The index $n$ gives the number of loopings between two reinjections for orbits leaving a strip $P^{(n)}$. As $\mu \rightarrow 0$, the segment $T B$ approaches $T_{\infty} B_{\infty}$ and thus increasingly more $P^{(n)}$ strips are allowed for the orbits. This process determines the permitted symbolic sequences. For example, in the $C^{(2)}$ regime of Fig. $4, M B_{2}$ crosses the strips $P^{(1)}, P^{(2)}$, and $P^{(3)}$; therefore a symbolic sequence $R(L)^{4}$ is incompatible with this situation. At the branch line of the template the semiflow induces a $1 \mathrm{D}$ return map $[5,8]$ that reflects

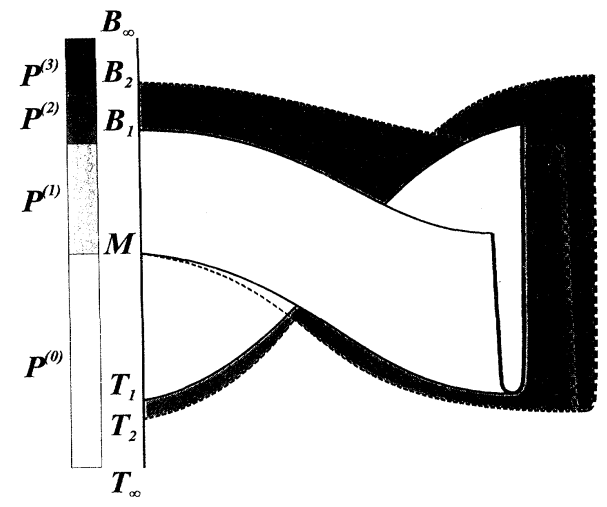

FIG. 4. Stretching and folding in the $C^{(1)}$ (white) and $C^{(2)}$ (gray) attractors. 
the metrics of the attractor when $\mu \rightarrow 0$. The transformation of this map under the change of $\mu$ accounts for the bifurcations in the APC sequence. As $\mu \rightarrow 0$, orbits of $P^{(n)}$ kind with increasingly higher period are generated and, having a correspondingly larger reinjection ordinate [8], they accumulate at $T_{\infty}$, which is also a homoclinic point because $T_{\infty}=f_{\mu=0}^{-1}\left(x_{T}, 0\right)$. Thus, $T_{\infty}$ is an accumulation point for an infinite family of periodic orbits according to a theorem by Birkhoff and Smith [16].

Although the template should remain invariant under small control parameter changes, we have also obtained experimental results that clearly evidence the alteration of the template, induced by a control parameter variation. These results show that the horseshoe template corresponds to the $C^{(1)}$, and the $C^{(2)}$ chaotic windows, while for the next window, $C^{(3)}$, a template with two orientation preserving branches plus two orientation reversing branches is associated. With our analysis, regarding Fig. 2, the transition of the two-branched to the fourbranched template is easily explained considering that the transformation of $T B$ under $f$, after the transition, extends the segment to the marked points (hollow dots) on $W^{u}(p)$ in Fig. 2 [17].

In conclusion, we have shown that homoclinic chaos in the GD is modeled by a horseshoe template. The template was obtained directly from the flow's geometry resulting from the formation of a homoclinic tangle. The analysis is independent on the elected embedding; we have obtained the same result for a differential phase space embedding [6]. We have made the analysis of homoclinic chaos through a topological approach instead of the usual technique employing return maps for analyzing the orbit structure near homoclinic orbits [18]. Both approaches, regarding the information obtained from the symbolic dynamics, are equivalent. But our topological analysis achieves a straightforward interpretation of the dynamical alterations in the GD which are reflected in the change of the template. In this situation, the approach based on maps presents the problem that the description passes from a simple 1D map to a troublesome $2 \mathrm{D}$ map. Our analysis is noise robust and there is no need of an explicit knowledge of the GD model equations. Therefore the procedure outlined in this paper is also applicable to other dynamical systems, provided they have a faithful phase space representation.

We acknowledge financial support from the Brazilian agencies CNPq, FINEP, and FAPERGS. T.B. is greatly indebted to N. B. Abraham, E. Arimondo, and P. M. Mors.
[1] H. Poincaré, Les Méthodes Nouvelles de la Mécanique Céleste (Gauthier-Villars, Paris, 1899), Vols. 1-3.

[2] J. Guckenheimer and P. Holmes, Nonlinear Oscillations, Dynamical Systems, and Bifurcations of Vector Fields (Springer-Verlag, New York, 1983).

[3] H. D. I. Abarbanel, R. Brown, J. J. Sidrowich, and L. S. Tsimring, Rev. Mod. Phys. 65, 1331 (1993).

[4] J. S. Birman and R. F. Williams, Topology 22, 47 (1983).

[5] P. Holmes, in New Directions in Dynamical Systems, edited by T. Bedford and J. Swift (Cambridge University Press, Cambridge, 1988), p. 150; P. J. Holmes and R. F. Williams, Arch. Ration. Mech. Anal. 90, 115 (1985).

[6] For an introductory account on the subject of templates, see G. B. Mindlin and R. Gilmore, Physica D 58, 229 (1992), N. B. Tufillaro, T. A. Abbott, and J. P. Reilly, An Experimental Approach to Nonlinear Dynamics and Chaos (Addison-Wesley, New York, 1992), Chap. 5.

[7] T. Braun, J A. Lisboa, and J. A. C. Gallas, Phys. Rev. Lett. 68, 2770 (1992).

[8] T. Braun and J. A. Lisboa, Int. J. Bifurc. Chaos (to be published).

[9] D. P. Lathrop and E. J. Kostelich, Phys. Rev. A 40, 4028 (1989).

[10] N. K. Gavrilov and L. P. Shilnikov, Math. USSR Sbornik 17, 467 (1972); 19, 139 (1973).

[11] M. Lefranc, D. Hennequin, and D. Dangoisse, J. Opt. Soc. Am. B 8, 239 (1991); F. Papoff, A. Fioretti, and E. Arimondo, Phys. Rev. A 44, 4639 (1991).

[12] J. W. L. McCallum and R. Gilmore, Int. J. Bifurc. Chaos 3, 685 (1993).

[13] M. Lefranc and P. Glorieux, Int. J. Bifurc. Chaos 3, 643 (1993).

[14] E. Arimondo and F. Molesti (private communication).

[15] F. Papoff, A. Fioretti, E. Arimondo, G. B. Mindlin, H. G. Solari, and R. Gilmore, Phys. Rev. Lett. 68, 1128 (1992).

[16] R. Abraham and C. Shaw, Dynamics - the Geometry of Behavior (Aerial Press, Santa Cruz, 1988), Vol. 3.

[17] T. Braun and R. R. B. Correia (unpublished).

[18] S. Wiggins, Introduction to Applied Nonlinear Dynamical Systems and Chaos (Springer-Verlag, New York, 1990). 


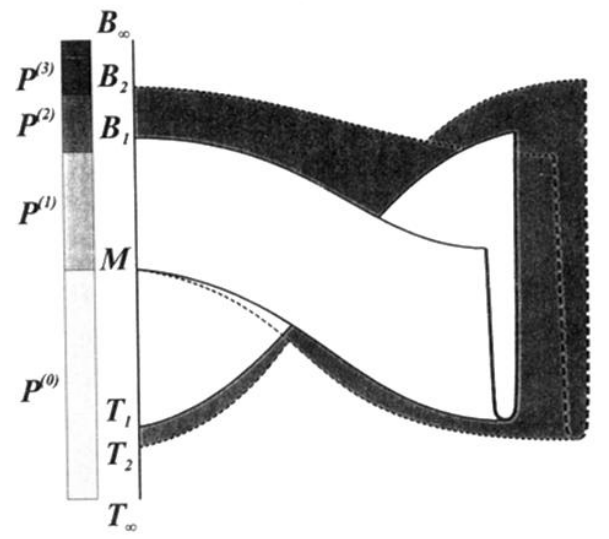

FIG. 4. Stretching and folding in the $C^{(1)}$ (white) and $C^{(2)}$ (gray) attractors. 